\title{
Mechanical Characterization of PDMS Films for the Optimization of Polymer Based Flexible Capacitive Pressure Microsensors
}

\author{
T.-H.-N. Dinh, E. Martincic, E. Dufour-Gergam, and P.-Y. Joubert \\ Centre of Nanosciences and Nanotechnologies (C2N-Orsay), CNRS, Université Paris Sud, Université Paris-Saclay, Orsay, France
}

Correspondence should be addressed to T.-H.-N. Dinh; thi-hong-nhung.dinh@u-psud.fr

Received 23 January 2017; Accepted 23 March 2017; Published 12 April 2017

Academic Editor: Zubair Ahmad

Copyright (C) 2017 T.-H.-N. Dinh et al. This is an open access article distributed under the Creative Commons Attribution License, which permits unrestricted use, distribution, and reproduction in any medium, provided the original work is properly cited.

\begin{abstract}
This paper reports on the optimization of flexible PDMS-based normal pressure capacitive microsensors dedicated to wearable applications. The operating principle and the fabrication process of such microsensors are presented. Then, the deformations under local pressure of PDMS thin films of thicknesses ranging from $100 \mu \mathrm{m}$ to $10 \mathrm{~mm}$ are studied by means of numerical simulations in order to foresee the sensitivity of the considered microsensors. The study points out that, for a given PDMS type, the sensor form ratio plays a major role in its sensitivity. Indeed, for a given PDMS film, the expected capacitance change under a $10 \mathrm{~N}$ load applied on a $1.7 \mathrm{~mm}$ radius electrode varies from a few percent to almost $40 \%$ according to the initial PDMS film thickness. These observations are validated by experimental characterizations carried out on PDMS film samples of various thicknesses ( $10 \mu \mathrm{m}$ to $10 \mathrm{~mm}$ ) and on actual microsensors. Further computations enable generalized sensor design rules to be highlighted. Considering practical limitations in the fabrication and in the implementation of the actual microsensors, design rules based on computed form ratio optimization lead to the elaboration of flexible pressure microsensors exhibiting a sensitivity which reaches up to $10 \% / \mathrm{N}$.
\end{abstract}

\section{Introduction}

Thanks are due to progress made in the fabrication of flexible electronics and microsensors, wearable physiological measurement devices are more and more developed for ambulatory and self-monitoring purposes [1]. Among them, devices using polymer based capacitive microsensors are very popular since they gather advantageous features, such as being sensitive to various physiological quantities as well as being easily designed for wearable use. This kind of sensors is basically constituted of metallic electrodes deposited on or imbedded in dielectric polymer films. Among the existing polymers, polydimethylsiloxane (PDMS) is a primary reference material, hence suitable for wearable use [2], especially when coated with suitable highly biocompatible material [3, 4]. Besides, PDMS is a dielectric material [5]; it is appropriate to be used in capacitive sensing applications. Indeed, its dielectric constant is considered by the manufacturer to be varying from 2.68 up to 2.72 according to the frequency of the used electrical field. In addition, for a given frequency, the permittivity of the PDMS can be determined with a $0.5 \%$ accuracy [5]. Besides, PDMS films feature very interesting mechanical behavior. Massive PDMS bulks are homogenous and isotropic and are highly deformable. They are able to undergo 100\% deformations and to come back to their original shape without any damage [6]. In addition, they are robust to mechanical stress, and rather insensitive to fatigue and temperature changes $[7,8]$.

These features allow flexible devices and sensing devices based on mechanical deformations to be designed and produced. As a result, PDMS films are therefore particularly well suited to be part of wearable capacitive force or pressure sensors [9]. Indeed, associated with metallic electrodes, the deformation of the PDMS films under pressure induces capacitance changes between electrodes that can be read out for pressure measurement purposes. For that reason, PDMSbased capacitive sensors are found in many medical applications involving pressure monitoring, such as implanted pressure sensors [4], tactile sensing [10,11], gesture, or gait analysis [12]. Since PDMS films are deformable under shear 
and normal forces, three-axis pressure capacitive microsensor arrays can be developed with appropriate electrode design [13]. However, it has been shown that the thickness of a PDMS film as well as the area on which the load is actually applied can strongly affect its deformation properties and hence the resulting capacitance change $[14,15]$. Besides, PDMS thin films are known to be hardly compressible, with a Poisson coefficient close to 0.49 [16]. This is why capacitive normal pressure sensors constituted of homogeneous PDMS films are often reported as lacking sensitivity to normal pressure (the sensitivity generally observed is around $1-2 \% / \mathrm{N}$ in a $10 \mathrm{~N}$ measurement range) $[13,17]$. Hence authors have proposed devices using heterogeneous PDMS films (e.g., featuring inner cavities) to enhance the sensor sensitivity to normal pressure [17]. However the fabrication processes associated with these designs are more complex and can result in higher manufacturing costs. In addition, the obtained structures make it even more difficult to foresee or adjust the sensitivity and the measurement range of the fabricated sensors.

This paper focuses on the design and the optimization of pressure microsensors dedicated to the development of wearable sensing devices, using homogeneous PDMS thin films. In order to accurately determine adequate sensor design and optimization rules, it is necessary to thoroughly study the mechanical behavior of PDMF thin films. Indeed, these mechanical properties are not easily predictable. Firstly, it is established that the macroscopic mechanical behavior of the fabricated PDMS films largely depends on the fabrication conditions, such as the weight ratio of prepolymer/curing agent used to fabricate the PDMS film or the intensity and the duration of the implemented thermal process. Indeed, Young's modulus $E$ is usually reported to be ranging from $500 \mathrm{kPa}$ to $3 \mathrm{MPa}$ and shear modulus $G$ from $400 \mathrm{kPa}$ et $900 \mathrm{kPa}$, according to the fabrication parameters $[15,16]$. Moreover, these properties are strongly dependent on the thickness of the fabricated PDMS film. For example, the elasticity of a given film type has been reported as being nonlinearly dependent on the thickness of the fabricated film $[9,15]$. Finally, PDMS films are hardly compressible (Poisson coefficient $v$ close to 0.5 ); the accurate knowledge of the mechanical behavior of thin films under local stresses is of considerable importance for the appropriate design of normal pressure microsensors.

The paper is organized as follows. First, in Section 2, the basic principle and constitution of the considered microsensors are presented, together with a general overview of its fabrication process. Then, Section 3 is dedicated to the numerical computations which are implemented to explore the behavior of PDMS thin films under local stress, for known macroscopic mechanical parameters of the films. In Section 4, experimental characterizations of fabricated PDMS thin films are carried out to validate the computational study, and then sensor design and optimization rules are highlighted. In Section 5, fabricated microsensors are electromechanically characterized and the obtained sensing performances are compared to the expected ones. Also, design rules are pointed out considering the practical limitations due to the fabrication and the implementation of the designed microsensors. Section 6 is on conclusions.

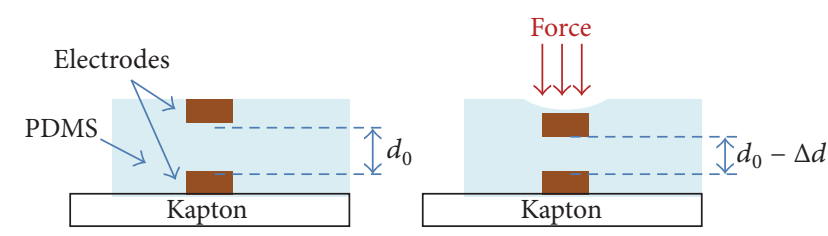

FIGURE 1: Cut view of a single planar capacitive sensor dedicated to normal pressure sensing.

\section{Basic Principle of the Sensor and Fabrication Process}

For the sake of simplicity, a simple parallel plate capacitive normal pressure microsensor is considered in this study. The basic principle of operation of such sensor is presented in Figure 1. The sensor is constituted of two square-plate copper electrodes facing one another. The electrodes are separated by a deformable dielectric PDMS thin film. The whole set is deposited on a Kapton substrate, used to provide both flexibility and mechanical robustness to the fabricated device. In load-free conditions, the capacitance of such sensor is given by [18]

$$
C=(2.47 b+1) \frac{\varepsilon_{0} \varepsilon_{r}}{d_{0}} l^{2},
$$

where $\varepsilon_{0}$ and $\varepsilon_{r}$ are the permittivity of vacuum and the dielectric constant of PDMS, respectively; $d_{0}$ is the distance between the electrodes; and $l$ is the electrode side length and $b=d_{0} / l$. The capacitance of the sensor is altered when a force is applied, since the distance $d_{0}$ between the two electrodes is changed from the quantity $\Delta d$. Reading out the capacitance change gives information about the force applied onto the surface the electrodes. Such microsensors dedicated to physiological pressure/force measurements are featured by millimetric dimensions for $l$ (typically $l=3 \mathrm{~mm}$ ) and micrometric to millimetric dimensions for $d_{0}$ (typically $d_{0}$ ranges from tens of micrometers up to a few millimetres) [19]. In order to fabricate PDMS-based pressure sensors, it is necessary to deposit copper electrodes at each side of PDMS films. It is however difficult to do so because of the low surface energy and of the highly compliant nature of PDMS films. To bypass this drawback, the so-called "filmtransfer" fabrication process is used. It enables assemblies using heterogeneous or even incompatible materials and technologies to be achieved. The fabrication procedure of arrays of such microsensors using film-transfer process has been fully detailed in [20].

Only the main fabrication steps are recalled here. The film-transfer process involves 4 fabrication steps (Figure 2). In the first step, copper top electrodes are realized on a donor wafer covered by a low adhesion layer and copper bottom electrodes are fabricated on a target wafer using the micromolding process (step (1)). Then, the fabricated copper lines are covered with PDMS (step (2)). It should be noted that a gap corresponding to the position of the conductor pad on the target substrate is intended for further electrical connection purposes. The third step aims at aligning and 
(1) Electroplated patterns by micromolding

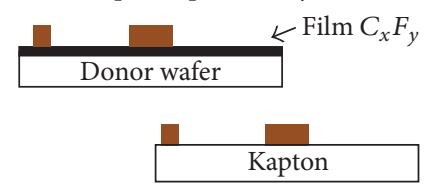

(3) Alignment and PDMS/PDMS bonding

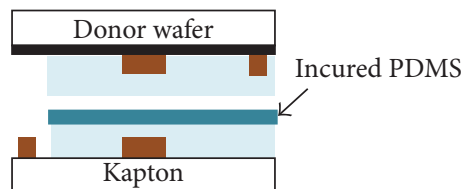

Copper

Cured PDMS
(2) PDMS spin coating

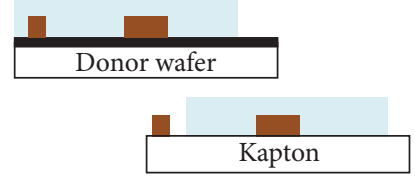

(4) Release of patterns from donor wafer

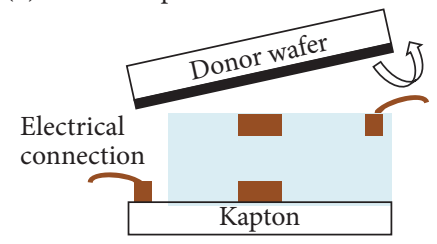

FIGURE 2: Four-step fabrication process of flexible microsensors.

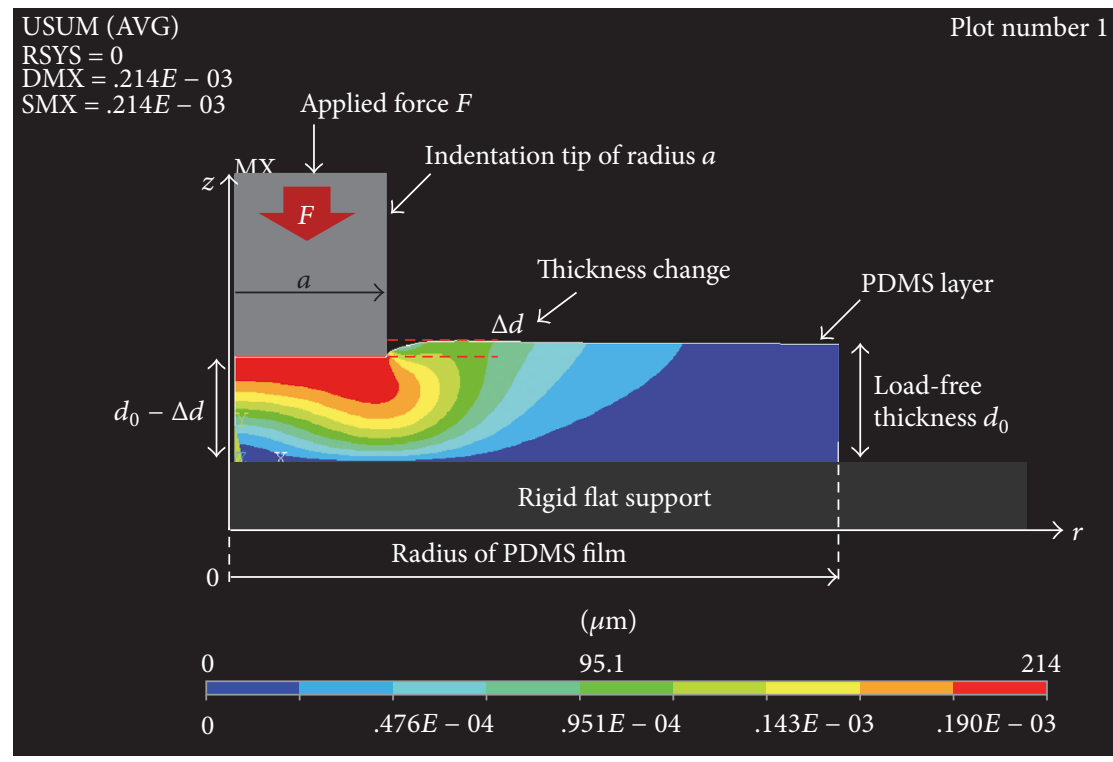

FIGURE 3: Finite element workspace for the computation of the mechanical behavior of PDMS films under load applied normally to its surface. Colors correspond to the magnitude of the displacement (in $\mu \mathrm{m}$ ).

bonding the two wafers using an uncured PDMS layer as glue. Finally, after removing the donor wafer, a capacitor with a PDMS dielectric layer is obtained (step (4)). Several sensor samples featuring various electrode sizes and PDMS film thicknesses have been successfully realized using this fabrication procedure. Some of the realized sensor samples are electromechanically characterized, as presented in Section 5 .

\section{Simulation of the Mechanical Behavior of PDMS Thin Films}

In this study, Sylgard 184 PDMS films are considered. They feature a prepolymer/curing agent ratio of $10 / 1$ by weight, and they are cured at $75^{\circ} \mathrm{C}$ during 1 hour. During fabrication, the thickness of the film samples is controlled by the speed of spin, during the spin coating procedure [20]. The fabricated film samples have thicknesses ranging from $120 \mu \mathrm{m}$ to $9.8 \mathrm{~mm}$. Considering the nature and the dimensions of the fabricated PDMS films, the density of the films is expected to be $970 \mathrm{~kg} / \mathrm{m}^{3}$ and Young's modulus $E$ is expected to be in the 1 to $3 \mathrm{MPa}$ range [20].

In order to foresee the mechanical behavior of PDMSbased microsensors, numerical simulations using finite element (FE) computations were carried out using Ansys Academic Research 12.1, implemented with 8 node elements (PLANE183 [21]). The whole computation workspace can be defined using axisymetrical geometry. As depicted in Figure 3, a two-dimensional representation of the workspace is considered in the $(O, r, z)$ plane. The three-dimensional distribution of the quantities of interest can be deduced 
from the two-dimensional results by rotation around the $(O, z)$ revolution axis. The workspace includes a cylindrical indentation tip which applies predetermined loads normally to the surface of a large circular PDMS film sample deposited on a Kapton substrate. The computations aim at evaluating the displacement field within the PDMS film when the force is locally applied by means of the indentation tip.

PDMS thin films of $10 \mathrm{~mm}$ diameter and of thicknesses ranging from $10 \mu \mathrm{m}$ to $10 \mathrm{~mm}$ are considered in the computations. Young's modulus of the PDMS films is set to $E=1 \mathrm{MPa}$ to $3 \mathrm{MPa}$, and the density is set to $970 \mathrm{~kg} / \mathrm{m}^{3}$. As the PDMS is known to be almost incompressible, Poisson's ratio is set to $v=0.450$ to $v=0.499$. Indentation tips featuring a radius $a$ ranging from $1.7 \mathrm{~mm}$ to $3 \mathrm{~mm}$ are considered to apply a $10 \mathrm{~N}$ load to the films. Besides, the Kapton substrate and the indentation tip are considered to be absolutely rigid. The computations are carried out under a linear assumption; that is, the PDMS behaves as if it undergoes small deformations so that Hooke's law can be considered. Considering the material features and the dimensions of the workspace, the used computational model is elaborated with up to 7,100 elements.

Thickness changes $\Delta d$ under the indentation tip are evaluated for a $10 \mathrm{~N}$ normal load (Figure 3 ). The computation results obtained for PDMS films featured by $E=1.3 \mathrm{MPa}$ and $\nu=0.499$ are presented versus initial thickness $d_{0}$ in Figure 4, for four different tip radii ranging from $a=1.7$ to $a=3 \mathrm{~mm}$. One can note that the thickness change $\Delta d$ depends on the initial load-free thickness $d_{0}$ of the film and on the radius $a$ of the indentation tip. More precisely, three main areas of operation can be observed in Figure 5.

In the first area, denoted as Zone 1 and defined by $10 \mu \mathrm{m}<$ $d_{0}<100 \mu \mathrm{m}$, one can observe that the thickness change $\Delta d$ under load is rather small (less than $1 \mu \mathrm{m}$ ). It slowly increases with the values of $d_{0}$, and decreases with the radius of the indentation tip. Indeed, in this operation area, the films can be considered as very thin compared to the radius of the indentation tip. The PDMS is hardly compressible and thin; it is actually difficult for the material to be compressed or to "evacuate" from below the indenter while the compression force is applied. As a result, only small local thickness changes $\Delta d$ can occur. This is even more noticeable when the indenter tip radius increases. This configuration of "thin" film in compression has already been studied in [14], in which the thickness changes $\Delta d$ have been related to the applied force $F$, Young's modulus $E$, the initial thickness $d_{0}$, and the radius of indentation $a$ so that

$$
\Delta d=\frac{3 F d_{0}}{4 E \pi a^{2}} .
$$

Another area of operation denoted Zone 3 can be defined by $d_{0}>5 \mathrm{~mm}$ (Figure 4 ). It corresponds to the case in which the initial thickness $d_{0}$ is equivalent or larger than the indenter's radius $a$. In this zone, it can be observed that the thickness change $\Delta d$ is important and tends to be independent from $d_{0}$ for large values of $d_{0}$. This "saturating" behavior may be explained by the fact that when the compression force is locally applied to the film, the material is actually able to "evacuate" from below the indenter tip, independently from the initial thickness $d_{0}$, as long as $d_{0}$ is large enough. This "thick" film configuration was also described in [14] in which the thickness change $\Delta d$ does not depend on $d_{0}$ and is written as

$$
\Delta d=\frac{3 F}{8 E a} .
$$

The third area of operation, denoted as Zone 2, is an intermediate zone for which no analytical formula has been proposed in the literature. Numerical simulations show that in this zone the thickness change $\Delta d$ induced by compression is large and is strongly related to the initial thickness $d_{0}$ and to the tip radius $a$. This feature may be advantageously used for the enhanced design of pressure capacitive sensors. In order to validate this assertion we determine the capacitance change $\Delta C$, expressed in $\%$, which would result from the thickness changes induced by the applied load on a PDMSbased capacitive sensor:

$$
\Delta C_{\%}=100 \frac{\Delta d}{d_{0}-\Delta d},
$$

where $\Delta d$ is the thickness changes provided by the FE computations and $d_{0}$ is the load-free thickness of the PDMS layer. The capacitance changes $\Delta C_{\%}$ are presented in Figure 5 as a function of $d_{0}$ and for four different tip radii ranging from $a=1.7$ to $3 \mathrm{~mm}$.

One can note that, for the considered applied load $(F=$ $10 \mathrm{~N}$ ), the sensor capacitance change $\Delta d$ is less than $1 \%$ for $d_{0}<100 \mu \mathrm{m}$ (Zone 1), increases and reaches a maximum for $100 \mu \mathrm{m}<d_{0}<5 \mathrm{~mm}$ (Zone 2), and rapidly decreases for $d_{0}>$ $5 \mathrm{~mm}$ (Zone 3 ). These observations confirm that the design of sensors carried out in Zone 2 seems judicious to maximize the sensitivity of the sensor, for a given size of the electrodes (or indentation tip radius). More precisely, it can be observed that the capacitance change reaches a maximum value when the so-called form ratio $\mathrm{FR}$, defined by $\mathrm{FR}=d_{0} / a$, is such that $\mathrm{FR} \approx 1.45$ (Figure 5 ) for the considered PDMS films. As a result, for a given indentation radius, the capacitance change varies from a few percent to tens of percent according to the initial load-free film thickness $d_{0}$. This means that the sensitivity of the sensor can be significantly optimized according to the relevant choice of $d_{0}$ and $a$ while designing the sensor.

In order to generalize this result, we carried out the same kind of computations for various values of Young's modulus $(E=1 \mathrm{MPa}, 1.3 \mathrm{MPa}$, and $2 \mathrm{MPa})$ and for Poisson coefficients $v$ varying between 0.45 and 0.499 . The computations results are shown in Figure 6. From the obtained curves, one can conclude that, for any kind of PDMS films, there exists an optimal form ratio FR which maximizes the capacitance change under load. As previously stated, this optimal FR depends on the geometry of the sensor $\left(d_{0}, a\right)$; it also depends on the mechanical parameters $(E, \nu)$ of the involved PDMS film. In other words, the sensitivity of the sensor can be optimized by the appropriate choice of $\left(d_{0}, a\right)$, once the mechanical parameters $(E, v)$ of the involved PDMS films are known. 


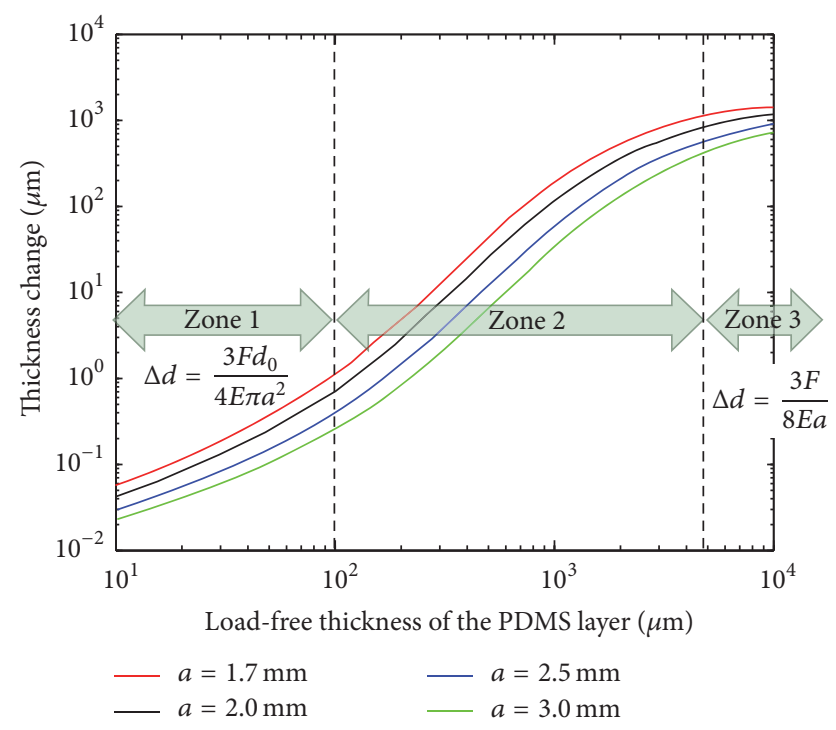

FIGURE 4: PDMS film thickness changes $\Delta d$ under a $10 \mathrm{~N}$ normal load, as a function of initial load-free thickness $d_{0}$ for indentation tips of various radii $a$.

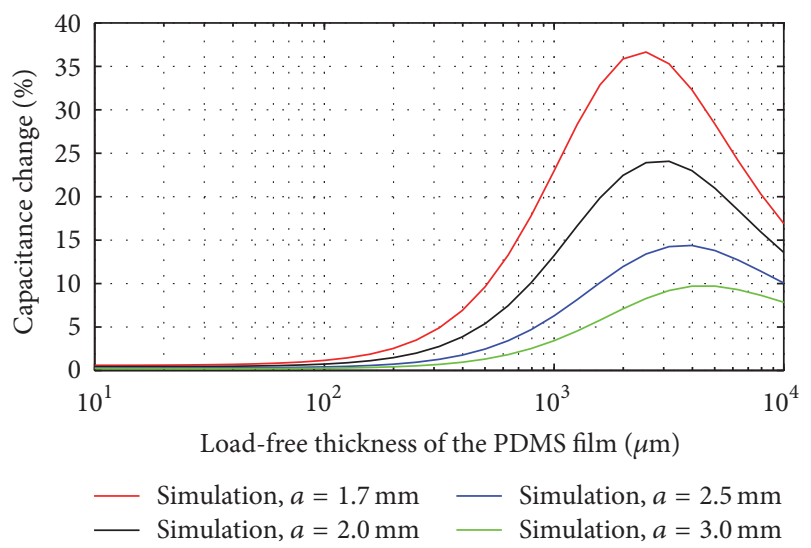

FIGURE 5: Capacitance changes induced by a $F=10 \mathrm{~N}$ force applied by an indenter tip of radius $a$, versus initial thickness $d_{0}$ of the used PDMS films.

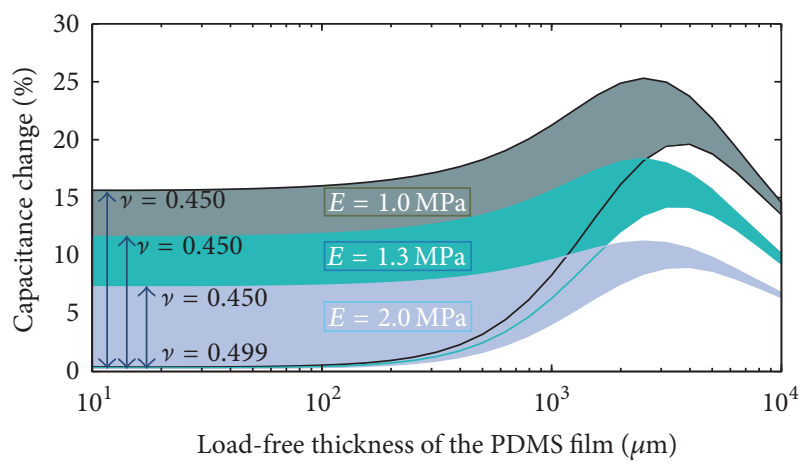

FIgURE 6: Capacitance changes induced by a $F=10 \mathrm{~N}$ force applied by an indenter tip of radius $a=2.5 \mathrm{~mm}$, for various values of $E$ and $\nu$, and versus initial thickness $d_{0}$ of the used PDMS films.

\section{Experimental Characterization of PDMS Thin Films}

The computations presented in the previous section were carried out under the assumptions that the PDMS films are homogenous and isotropic and that only small deformations are considered. To validate the results computed under these assumptions, $10 \mathrm{~cm}$ diameter PDMS films samples of various thicknesses $\left(120 \mu \mathrm{m}<d_{0}<9.8 \mathrm{~mm}\right)$ were experimentally characterized. To do so, a CSM Instruments microindentation tester is used (see Figure 7).

It is implemented with homemade cylindrical indentation tips of radius $a=2 \mathrm{~mm}$ and $a=2.5 \mathrm{~mm}$. The PDMS film sample is positioned under the indentation tip with a 3axis motorized table featuring a $0.25 \mu \mathrm{m}$ position resolution. The indentation tip, placed normally to the film surface, is driven within the sample with increasing loads ranging from 0 to $10 \mathrm{~N}$. The load is then gradually decreased until the complete relaxation of the film occurs. At each position of the indentation tip, the applied force is controlled and monitored with a $0.3 \mathrm{mN}$ resolution. The displacement of the tip is monitored with a $0.3 \mathrm{~nm}$ resolution.

The setup allows the thickness change $\Delta d$ to be evaluated as a function of the applied load. An example of the evaluation of $\Delta d$ versus applied load is given in Figure 8 in the case of a $3.04 \mathrm{~mm}$ thick PDMS film stressed with a $2.5 \mathrm{~mm}$ radius indentation tip. Same experimental characterizations are carried out for the whole set of PDMS film samples using the two indentation tips ( $a=2 \mathrm{~mm}$ and $2.5 \mathrm{~mm}$ ).

For each of the carried out experiments, the thickness change $\Delta d$ obtained under a $10 \mathrm{~N}$ load is stored and the according capacitance change $\Delta C_{\%}$ is determined using (4). The obtained experimental values are plotted in Figure 9, in addition to results computed with the same film dimensions and indenter tips and with $E=1.3 \mathrm{MPa}$ and $v=0.499$. One can note that a fair agreement is obtained between the experimental results and the simulated ones. The observed discrepancies may be attributed to inaccurate measurements of $d_{0}$ in the case of actual PDMS films, since the film thickness may not be perfectly constant across the whole surface of the film sample. These experiments validate the existence of an optimum form ratio FR which allows the capacitance change to be optimized knowing the mechanical properties of the used PDMS film.

\section{Application to the Design of Microsensors}

5.1. Electromechanical Characterization of Fabricated Microsensors. PDMS-based microsensors were actually fabricated on $10 \mathrm{~cm}$ diameter Kapton substrates by means of the fabrication process presented in Section 2. The used fabrication process enables single or multiple sensors together with their connection tracks to be fabricated with a transfer yield of $100 \%$ [20]. All the fabricated sensors feature $3 \mu \mathrm{m}$ thick copper electrodes. They are square shaped and feature $3 \times$ $3 \mathrm{~mm}^{2}$ electrodes. Six microsensors samples featuring PDMS thicknesses $d_{0}$ ranging from 43 to $660 \mu \mathrm{m}$ are fabricated, 


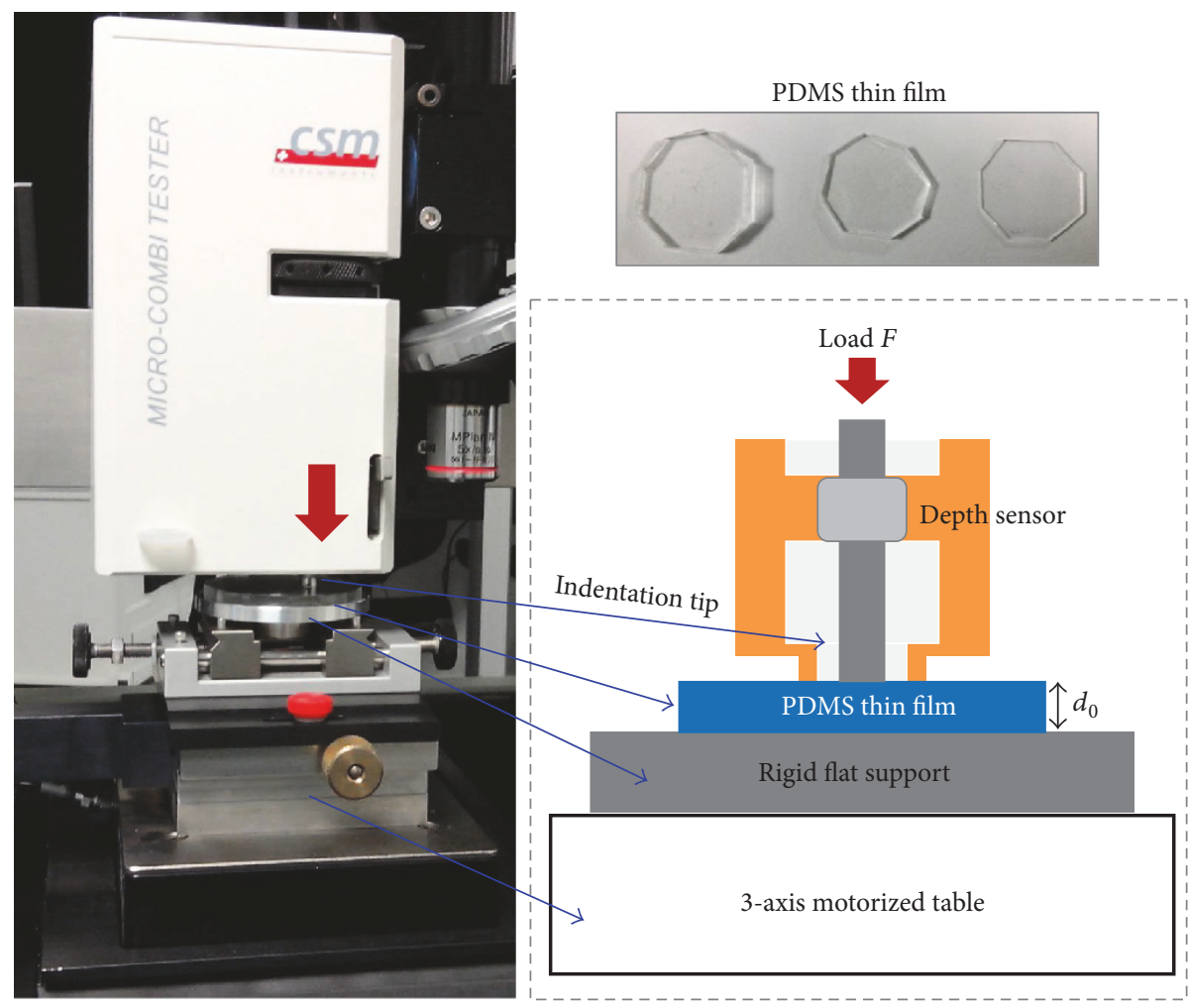

FIgURE 7: Indentation setup used for the mechanical characterization of PDMS films.

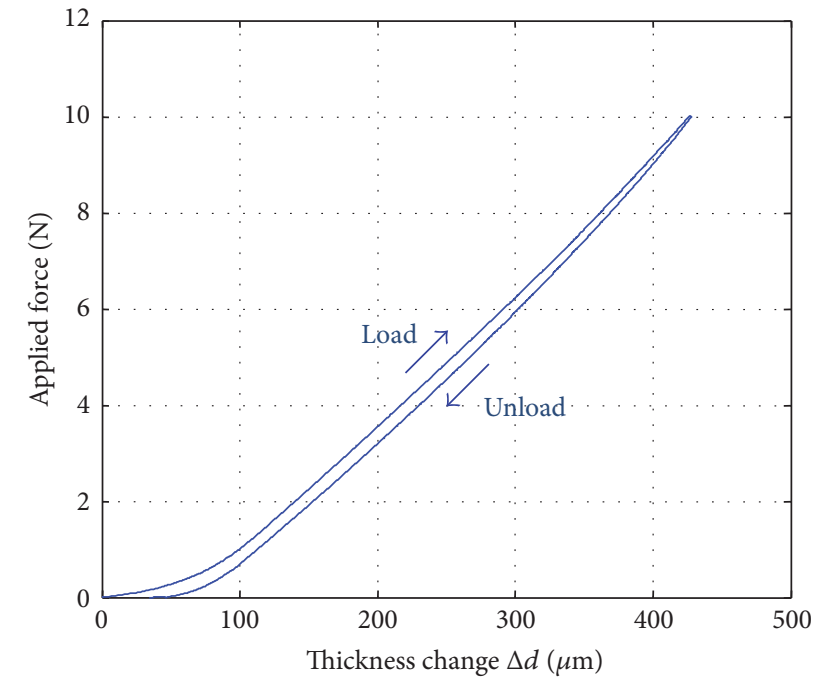

FIgURE 8: PDMS film thickness as a function of the applied load by a $a=2.5 \mathrm{~mm}$ radius indenter tip (initial thickness $d_{0}$ of the characterized PDMS film is $3.04 \mathrm{~mm}$ ).

so as to validate the importance of the form ratio FR on the capacitance change of the flexible microsensor under load. An example of a fabricated flexible sensor is shown in Figure 10(a) [22].

A dedicated electromechanical test bench was set up to carry out the electromechanical characterizations of the

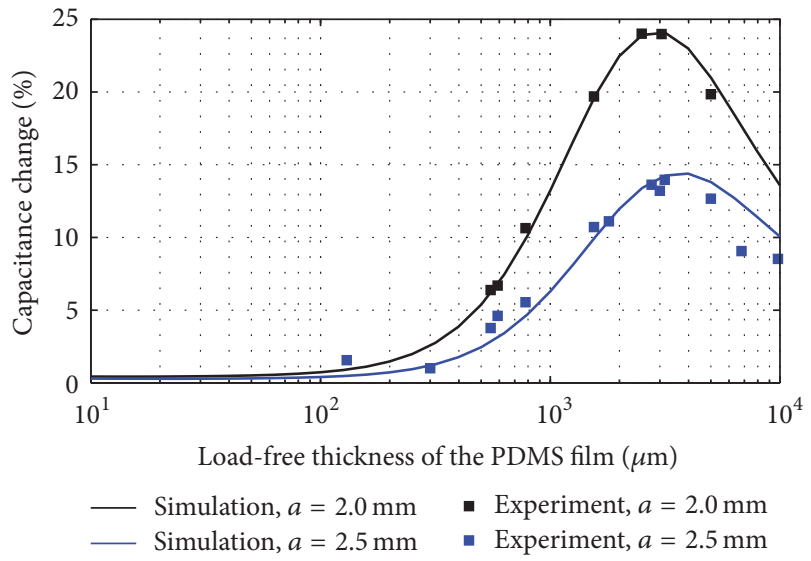

FIGURE 9: Evolution of the capacitance change under a $10 \mathrm{~N}$ load, obtained by computations $(E=1.3 \mathrm{MPa}$ and $v=0.499)$ and experimental characterizations.

fabricated microsensor samples (see Figure 10(b)). The setup includes an indenter tip associated with a spring loaded commercial force gauge, fixed on a PC controlled 3-axis robot $\mathrm{arm}$. The load applied to the capacitive microsensors is monitored using the commercial force gauge (Futek LSB200), and the capacitance changes are measured using a PC controlled HP4192A impedance analyzer. The capacitance changes of six microsensor samples (sensors 1 to 6) of various loadfree thicknesses measured for an applied force of $10 \mathrm{~N}$ are 
plotted in Figure 12, together with the simulated capacitance changes computed for the corresponding PDMS films. One can note that the actual capacitance changes of the fabricated microsensors are in fair agreement with the computed ones.

Since the electrodes of the actual microsensors are square shaped rather than circular, we define an equivalent electrode radius $a_{\mathrm{eq}}$ determined so that the surface of the square shaped electrodes and the equivalent circular shaped electrodes are equal:

$$
a_{\mathrm{eq}}=\frac{l}{\sqrt{\pi}},
$$

where $l$ is the side length of the square electrode. As a result, the fabricated microsensor samples feature form ratios $\mathrm{FR}=d_{0} / a_{\mathrm{eq}}$ which are varying from 0.03 to 0.39 , for $a_{\mathrm{eq}}=$ $1.7 \mathrm{~mm}$ and $d_{0}$ ranging from 43 to $660 \mu \mathrm{m}$. For the considered PDMS films and the indentation tip of radius $a=1.7 \mathrm{~mm}$, the optimal form ratio was computationally defined to be $\mathrm{FR} \approx 1.45$ (see Section 3 ). One can observe that the actual microsensors feature a capacitance change under $10 \mathrm{~N}$ load which ranges from $\Delta C=3 \%$ (sensor 1, designed with $\mathrm{FR}=$ 0.03 ) up to $\Delta C=17 \%$ (sensor 6 , designed with $\mathrm{FR}=$ 0.39 ), according to the FR value chosen for the design of the sensor. Indeed, sensor 6 exhibits the highest value of $\Delta C$, since it features the FR value which is closest to the optimum value $\mathrm{FR}=1.45$, comparatively to sensors 1 to 5 . These experimental results validate the role of the form ratio on the sensitivity of the design of microsensors.

5.2. Design Rules of PDMS-Based Flexible Microsensors. The previous sections highlighted the importance of the form ratio of the involved PDMS thin film on the capacitance change that can be expected from the loaded pressure sensor. The example presented in Figure 11 shows that a $3 \times 3 \mathrm{~mm}^{2}$ sensor exhibits capacitance changes under $10 \mathrm{~N}$ loads which can vary from a few percents to more than $35 \%$, according to the form ratio determined in the sensor design.

Computations can be carried out for a wider range of indentation radii (or electrode size) in order to extend the design possibilities. In Figure 12, capacitance changes computed for the same PDMS films $(E=1.3 \mathrm{MPa}$ and $\nu=0.499$ ) with indentation radii ranging from $3 \mathrm{~mm}$ down to $0.5 \mathrm{~mm}$ are plotted as a function of the initial load-free thickness $d_{0}$. One can note that the use of an indentation radius $a<1 \mathrm{~mm}$ allows capacitance changes $\Delta C>100 \%$ to be reached, thanks to the appropriate choice of $d_{0}$. This leads to many design possibilities allowing highly sensitive pressure microsensors to be designed.

However, in practice, design possibilities are constrained by three limitations. Firstly, sensor designs leading to PDMS deformations $\Delta d / d_{0}$ greater than $50 \%$ should be ruled out, since Hooke's law involving small deformations no longer applies, and computations lead to unrealistic results. Secondly, sensors designed with electrodes or initial thickness leading to too small values of the load-free capacitance $C$ should also be avoided for the sake of measurement accuracy (in practice, the lower limit is $C \approx 0.3 \mathrm{pF}$ using the HP4192A impedance analyzer). Thirdly, sensor designed with load-free thicknesses smaller than $d_{0}=25 \mu \mathrm{m}$ cannot be fabricated using the developed film-transfer process [20]. These three limitations are highlighted in the computation results presented in Figure 12. Nevertheless, one can note that a wide range of design possibilities remain available in practice, such as

(i) $a=1 \mathrm{~mm}$ and $d_{0}=400 \mu \mathrm{m}$, which leads to capacitance changes $\Delta C=60 \%$ under a $10 \mathrm{~N}$ load or a force sensing sensitivity of $6 \% / \mathrm{N}$;

(ii) $a=0.5 \mathrm{~mm}$ and $d_{0}=75 \mu \mathrm{m}$, which leads to capacitance changes $\Delta C=100 \%$ under a $10 \mathrm{~N}$ load or a force sensing sensitivity of $10 \% / \mathrm{N}$.

These examples show that PDMS-based capacitive microsensors providing sensitivities higher than generally observed for such sensors (around 2\%/N [13]) can be produced thanks to enhanced design.

\section{Conclusion}

This paper focuses on the design of flexible PDMS-based capacitive pressure microsensors dedicated to the wearable and noninvasive monitoring of pressure/force distributions required in some medical applications. A study of the mechanical behavior of PDMS thin films is carried out in order to foresee and optimize the performances of these sensors. First, a mechanical study of PDMS thin films is carried out using finite element computations as well as experimental characterizations by means of a microindentation tester. The study points out that the sensor sensitivity related to normal stress may be optimized according to the form ratio of the used PDMS thin film. For example, for a $9 \mathrm{~mm}^{2}$ sensor build up with a given PDMS film, a maximum capacitance change under a $10 \mathrm{~N}$ load theoretically reaches up to $37 \%$ when the optimized form ratio is used in the sensor design and reaches only a few percent otherwise. Such trends are validated by experimental electromechanical characterizations carried out on fabricated microsensors. Finally, further computations extended to a wider range of electrode sizes and load-free film thicknesses are made. Considering practical limitations in the fabrication and in the implementation of the actual microsensors, design rules based on computed form ratio optimizations should lead to the elaboration of flexible pressure microsensors exhibiting a sensitivity which reaches up to $10 \% / \mathrm{N}$. Further works should focus on the mechanical behavior of large PDMS films so that pressure microsensors arrays may be optimized. Furthermore, such study should be extended to the evaluation of the behavior of PDMS films to shear stress, in order to optimize 3 -axis pressure field distribution microsensor arrays based on the threedimensional deformation of PDMS films. Nevertheless, the presented study opens the way to the elaboration of enhanced PDMS-based pressure capacitive microsensors.

\section{Conflicts of Interest}

The authors declare that there are no conflicts of interest regarding the publication of this paper. 


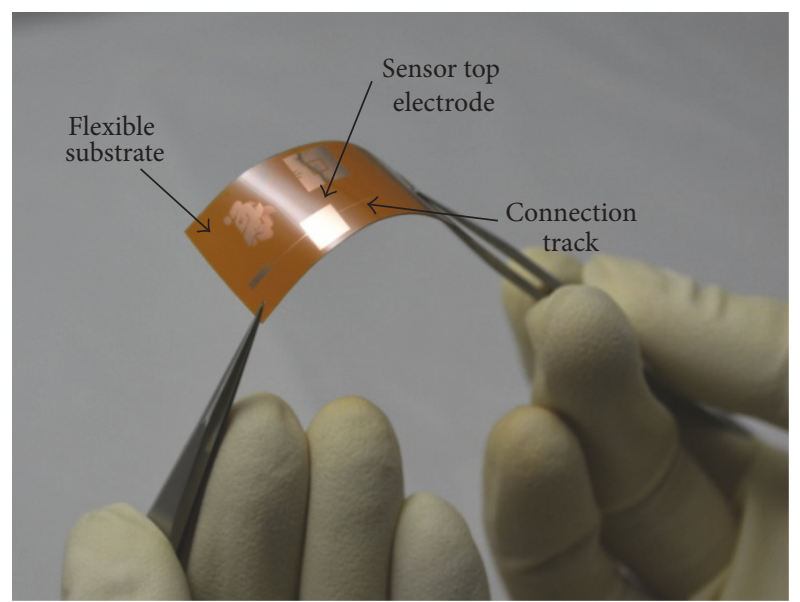

(a)

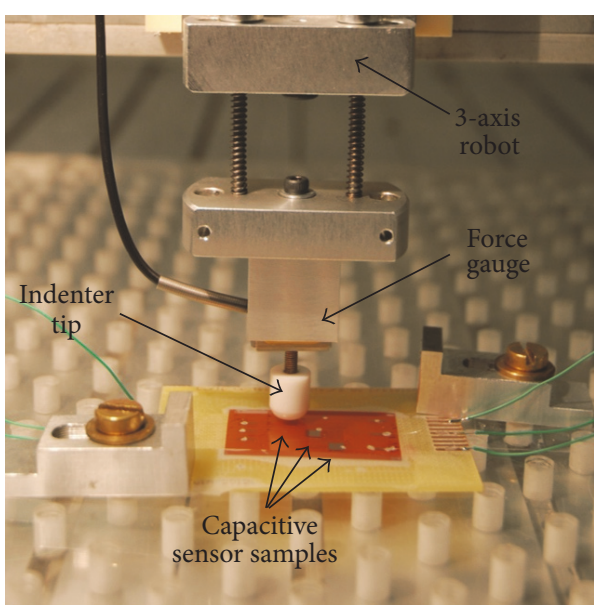

(b)

FIGURE 10: Flexible capacitive pressure sensor sample (a) and electromechanical characterization setup (b).

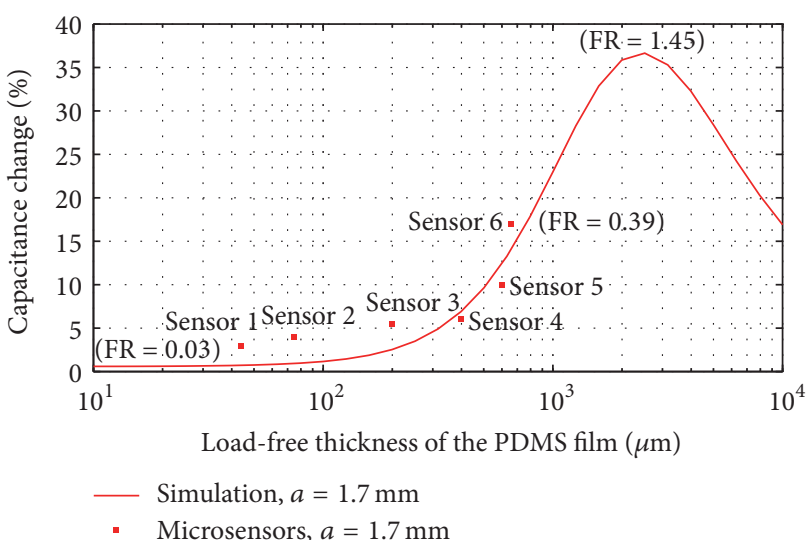

FIGURE 11: Microsensor capacitance changes obtained by experimental characterizations and corresponding capacitance changes obtained by simulations, as a function of PDMS thickness $d_{0}$ and electrode radius $a$, for and applied load of $10 \mathrm{~N}$.

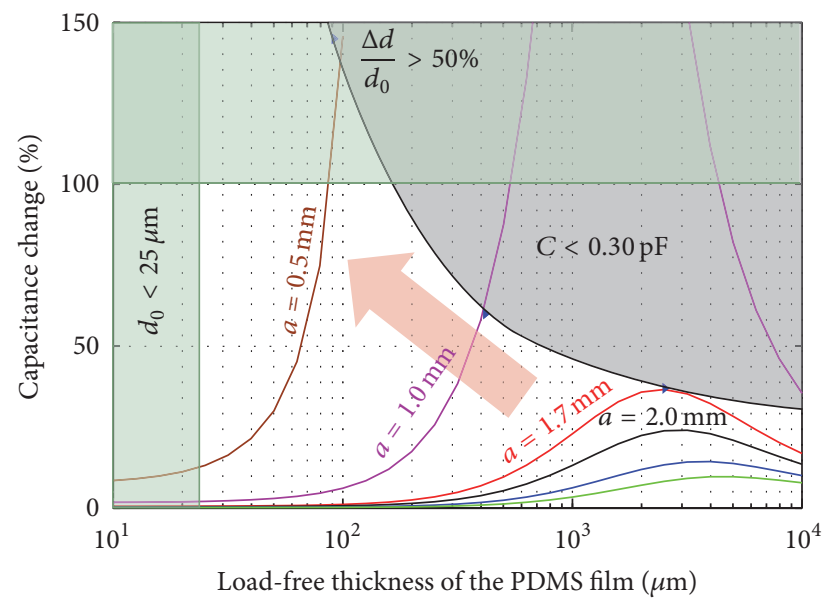

FIGURE 12: Design range allowing enhanced sensitivity capacitive microsensors to be produced.

\section{Acknowledgments}

This work has benefited from the financial support of the LabeX LaSIPS (ANR-10-LABX-0040-LaSIPS) managed by the French National Research Agency under the "Investissements d'Avenir" Program (no. ANR-11-IDEX-0003-02). The authors wish to thank technical staff of the clean room facilities of the "Centrale de Technologie Universitaire (MINERVE-CTU)" for its valuable help during the experimental tests.

\section{References}

[1] S. Patel, H. Park, P. Bonato, L. Chan, and M. Rodgers, "A review of wearable sensors and systems with application in rehabilitation," Journal of NeuroEngineering and Rehabilitation, vol. 9, no. 1, article 21, 2012.

[2] Y. Marois and M. C. Be, "Studies of primary reference materials low-density polyethylene and polydimethylsiloxane: a review," Journal of Biomedical Materials Research, vol. 58, pp. 467-477, 2001.

[3] M. Couty, A. Rubin, M. Woytasik et al., "Implantable wireless microcoils for 7Tesla Magnetic Resonance Imaging of the rat brain: optimization of the PDMS packaging," in Proceedings of the IEEE Topical Conference on Biomedical Wireless Technologies, Networks, and Sensing Systems (BioWireleSS '12), pp. 37-40, January 2012.

[4] D. Ha, W. N. de Vries, S. W. M. John, P. P. Irazoqui, and W. J. Chappell, "Polymer-based miniature flexible capacitive pressure sensor for intraocular pressure (IOP) monitoring inside a mouse eye," Biomedical Microdevices, vol. 14, no. 1, pp. 207-215, 2012.

[5] N. Tiercelin, P. Coquet, R. Sauleau, V. Senez, and H. Fujita, "Polydimethylsiloxane membranes for millimeter-wave planar ultra flexible antennas," Journal of Micromechanics and Microengineering, vol. 16, no. 11, pp. 2389-2395, 2006.

[6] F. Schneider, T. Fellner, J. Wilde, and U. Wallrabe, "Mechanical properties of silicones for MEMS," Journal of Micromechanics and Microengineering, vol. 18, no. 6, Article ID 065008, 2008. 
[7] A. Mata, A. J. Fleischman, and S. Roy, "Characterization of polydimethylsiloxane (PDMS) properties for biomedical micro/nanosystems," Biomedical Microdevices, vol. 7, no. 4, pp. 281-293, 2005.

[8] J. C. Lötters, W. Olthuis, P. H. Veltink, and P. Bergveld, "The mechanical properties of the rubber elastic polymer polydimethylsiloxane for sensor applications," Journal of Micromechanics and Microengineering, vol. 7, no. 3, pp. 145-147, 1997.

[9] S. El-Molla, A. Albrecht, E. Cagatay et al., "Integration of a thin film PDMS-based capacitive sensor for tactile sensing in an electronic skin," Journal of Sensors, vol. 2016, Article ID 1736169, 7 pages, 2016.

[10] A. M. Almassri, W. Z. W. Hasan, S. A. Ahmad et al., "Pressure sensor: state of the art, design, and application for robotic hand," Journal of Sensors, vol. 2015, Article ID 846487, 12 pages, 2015.

[11] M. I. Tiwana, S. J. Redmond, and N. H. Lovell, "A review of tactile sensing technologies with applications in biomedical engineering," Sensors and Actuators, A: Physical, vol. 179, pp. 1731, 2012.

[12] K. F. Lei, K.-F. Lee, and M.-Y. Lee, "A flexible PDMS capacitive tactile sensor with adjustable measurement range for plantar pressure measurement," Microsystem Technologies, vol. 20, no. 7, pp. 1351-1358, 2014.

[13] R. Surapaneni, Q. Guo, Y. Xie, D. J. Young, and C. H. Mastrangelo, "A three-axis high-resolution capacitive tactile imager system based on floating comb electrodes," Journal of Micromechanics and Microengineering, vol. 23, no. 7, Article ID 075004, 2013.

[14] F. Yang, "Thickness effect on the indentation of an elastic layer," Materials Science and Engineering A, vol. 358, no. 1-2, pp. 226232, 2003

[15] M. Liu, J. Sun, Y. Sun, C. Bock, and Q. Chen, "Thicknessdependent mechanical properties of polydimethylsiloxane membranes," Journal of Micromechanics and Microengineering, vol. 19, no. 3, Article ID 035028, 2009.

[16] I. D. Johnston, D. K. McCluskey, C. K. L. Tan, and M. C. Tracey, "Mechanical characterization of bulk Sylgard 184 for microfluidics and microengineering," Journal of Micromechanics and Microengineering, vol. 24, no. 3, Article ID 035017, 2014.

[17] S. C. B. Mannsfeld, B. C.-K. Tee, R. M. Stoltenberg et al., "Highly sensitive flexible pressure sensors with microstructured rubber dielectric layers," Nature Materials, vol. 9, no. 10, pp. 859-864, 2010.

[18] H. Nishiyama, "Form and capacitance of parallel-plate capacitors," IEEE Transactions on Components Packaging and Manufacturing Technology Part A, vol. 17, no. 3, pp. 477-484, 1994.

[19] T. H. N. Dinh, P.-Y. Joubert, E. Martincic, and E. DufourGergam, "Polymer-based flexible capacitive pressure sensor for non-invasive medical monitoring applications," in Proceedings of the 9th IEEE International Symposium on Medical Measurements and Applications (IEEE MeMeA '14), vol. 8622, pp. 270274, Lisbon, Portugal, June 2014.

[20] T. H. N. Dinh, E. Martincic, E. Dufour-Gergam, and P.-Y. Joubert, "Capacitive flexible pressure sensor: microfabrication process and experimental characterization," Microsystem Technologies, vol. 22, no. 3, pp. 465-471, 2016.

[21] T. D. Canonsburg, ANSYS Mechanical User Guide, vol. 15317, 2012.

[22] T. H. N. Dinh, E. Martincic, E. Dufour-Gergam, and P.-Y. Joubert, "Experimental study of PDMS mechanical properties for the optimization of polymer based flexible pressure microsensors," Journal of Physics: Conference Series, vol. 757, Article ID 012009, 2016 


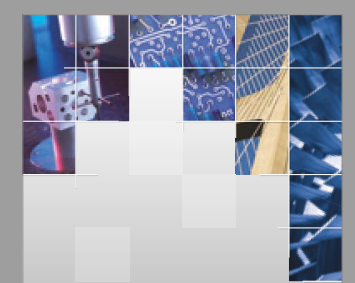

\section{Enfincering}
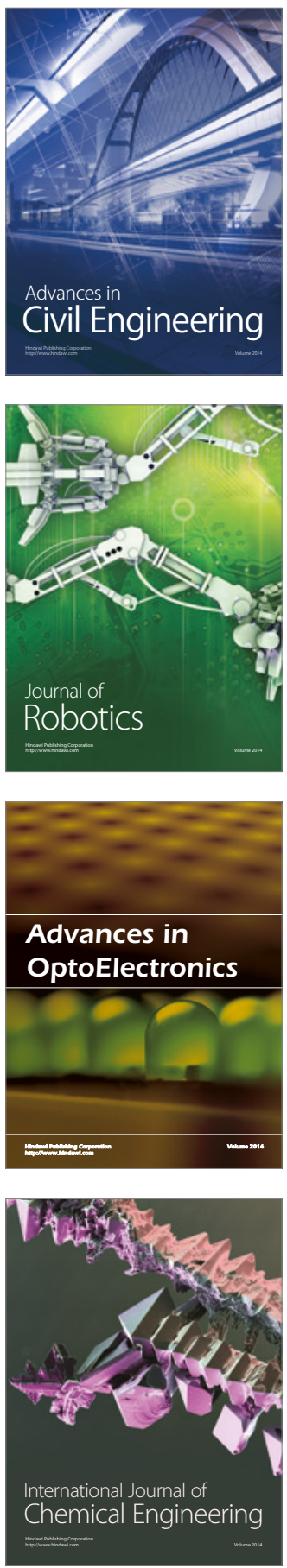

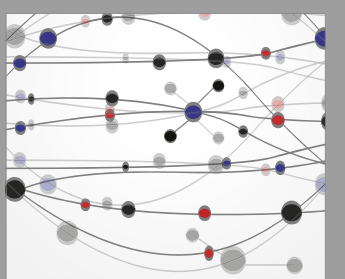

The Scientific World Journal

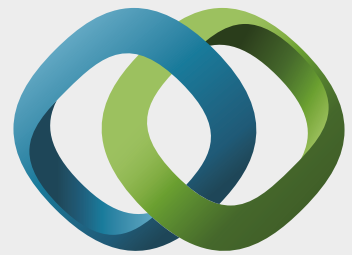

\section{Hindawi}

Submit your manuscripts at

https://www.hindawi.com
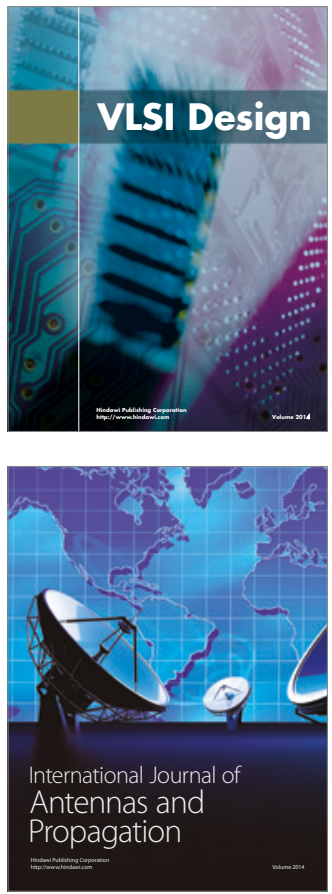

\section{Rotating}

Machinery
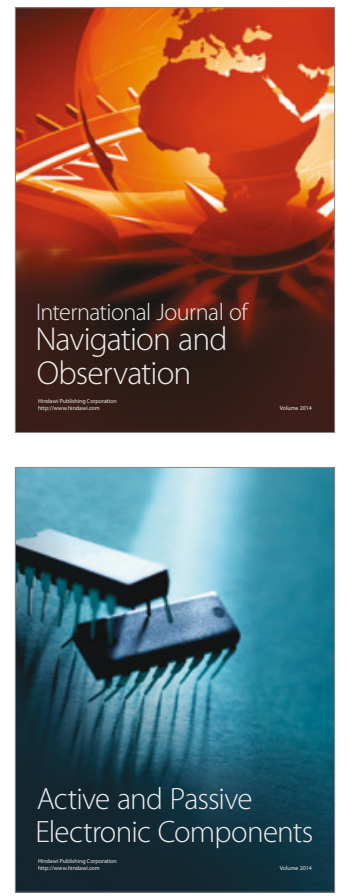
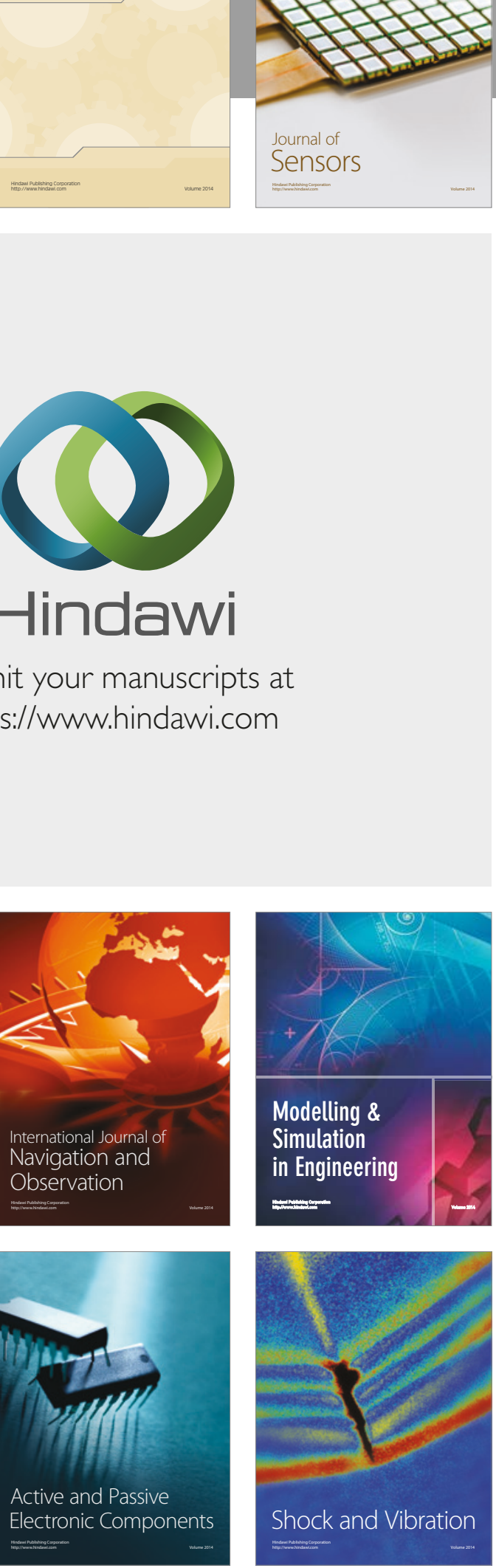
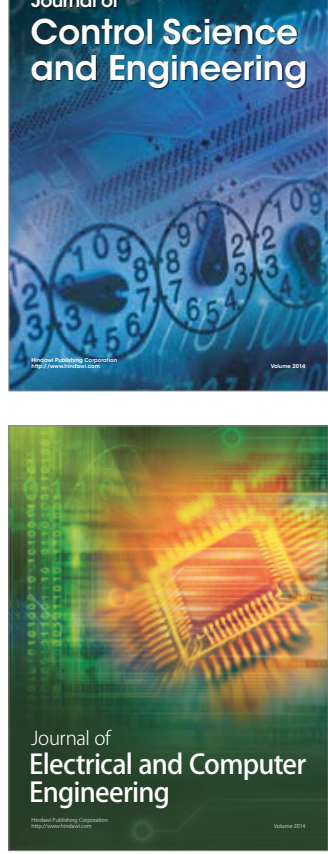

Distributed

Journal of

Control Science

and Engineering
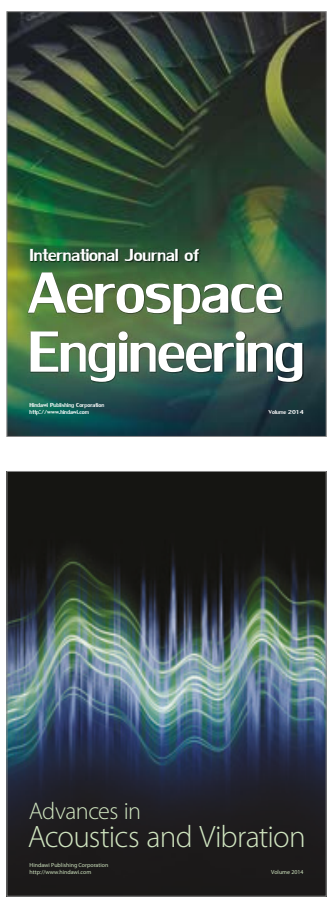

Sensor Networks 